\title{
PROCESSO DE ENFERMAGEM: REFLEXÕES DE AUXILIARES E TÉCNICOS ${ }^{1}$ NURSING PROCESS: REFLECTIONS OF AIDES AND TECHNICIANS EL PROCESO DE ENFERMERÍA: REFLEXIONES DE AUXILIARES Y TÉCNICOS
}

\author{
Vanessa Kenne Longaray², Miriam de Abreu Almeida ${ }^{3}$ Paula de Cezaro ${ }^{4}$
}

\footnotetext{
${ }^{1}$ Estudo extraído do Trabalho de Conclusão de Curso "Processo de Enfermagem-Percepções de Técnicos e Auxiliares de Enfermagem", apresentado ao Curso de Enfermagem da Universidade Federal do Rio Grande do Sul (UFRGS).

${ }^{2}$ Enfermeira do Hospital de Clínicas de Porto Alegre. Rio Grande do Sul, Brasil.

${ }^{3}$ Doutora em Educação. Professora Adjunto da Escola de Enfermagem da UFRGS. Rio Grande do Sul, Brasil.

${ }^{4}$ Enfermeira Residente do Programa Residência Integrada em Saúde do Grupo Hospital Conceição com ênfase em Terapia Intensiva. Rio Grande do Sul, Brasil.
}

PALAVRAS-CHAVE: Enfermagem. Processos de enfermagem. Equipe de enfermagem. Assistência hospitalar. Cuidadores.
RESUMO: O Processo de Enfermagem consiste em um instrumento tecnológico empregado para favorecer o cuidado. Trata-se de uma pesquisa qualitativa exploratória descritiva realizada no primeiro semestre de 2006, com o objetivo de conhecer as reflexões de técnicos e auxiliares sobre o Processo de Enfermagem, no Hospital de Clínicas de Porto Alegre - RS. Participaram da pesquisa 11 sujeitos de diferentes serviços. Utilizou-se entrevista semi-estruturada para obtenção de informações e análise temática de Bardin. Na análise identificaram-se cinco categorias: 1) amplia a visão e organiza o cuidado; 2) aproxima a equipe de enfermagem;3) favorece a continuidade dos cuidados;4) falhas na comunicação entre a equipe de enfermagem; 5) necessidade de reavaliação da prescrição dos cuidados de enfermagem. Diante do exposto sugere-se fomentar educação em serviço para a equipe de enfermagem tendo como foco o Processo de Enfermagem, dando-se ênfase à comunicação.
KEYWORDS: Nursing. Nursing process. Team nursing. Hospital care. Caregivers.
ABSTRACT: The Nursing Process consists of a technological instrument employed to favor care. This study is a descriptive, exploratory, qualitative research carried through in the first half of 2006. The main objective of this study is to better understand the reflections of nursing technicians and aides concerning the Nursing Process carried out in the Hospital de Clínicas in Porto Alegre, RS, Brazil. Eleven subjects from different services participated in the research. Semi-structured interviews were used to obtain the data and apply the Bardin thematic analysis. Five categories were identified in the analysis: 1) amplifying vision and organizing care; 2 ) bringing the Nursing team closer; 3 ) favoring care favoring; 4) communication failures among the nursing team; 5) the need for reevaluating the prescription of nursing care. It is suggested, based on these observations, to promote in-service education for the nursing team, focusing on the Nursing Process and emphasizing communication.
PALABRAS CLAVE: Enfermería. Procesos de enfermería. Grupo de enfermería. Atención hospitalaria. Cuidadores.
RESUMEN: El Proceso de Enfermería consiste en un instrumento tecnológico empleado para favorecer el cuidado. El presente estudio trata de una investigación cualitativa, exploratoria y descriptiva, realizada en el primer semestre de 2006, con el objetivo de conocer las reflexiones de técnicos y auxiliares sobre el Proceso de Enfermería realizado en el Hospital de Clínicas de Porto Alegre - RS. De la investigación participaron 11 sujetos pertenecientes a distintos servicios. Para la obtención de las informaciones y el análisis temático de Bardin, se utilizó la entrevista parcialmente estructurada. En el análisis fueron identificadas cinco categorías, a saber: 1) amplía la visión y organiza el cuidado; 2) aproxima el equipo de enfermería; 3) favorece la continuidad de los cuidados; 4) fallas en la comunicación entre el equipo de enfermería; 5) necesidad de revaluar la prescripción de los cuidados de enfermería. Ante lo expuesto, se sugiere fomentar la educación en el servicio para el equipo de enfermería, teniendo como foco el Proceso de Enfermería y dando énfasis a la comunicación.
Vanessa Kenne Longaray Endereço: R. Ovideo Moraes Leal, 60 91.180-110 - Porto Alegre, RS, Brasil. E-mail: nessa_kl@yahoo.com.br
Artigo original: Pesquisa Recebido em: 16 de julho de 2007 Aprovação final: 15 de janeiro de 2008 


\section{INTRODUÇÃO}

O Processo de Enfermagem (PE) consiste em um instrumento metodológico empregado para favorecer o cuidado, além de organizar as condições necessárias para a sua ocorrência. As etapas que o compõem - diagnóstico, intervenções e resultados de enfermagem - contribuem para o desenvolvimento de sistemas de classificação, considerados instrumentos tecnológicos importantes para o processo de raciocínio e julgamento clínico dos elementos da prática profissional das enfermeiras. ${ }^{1}$

Nos anos 70, a metodologia do PE invadiu as escolas de enfermagem brasileiras, a partir das contribuições de Wanda Horta. ${ }^{2}$ Ainda nesta década, o PE foi inserido no ensino de Graduação da Escola de Enfermagem da Universidade do Rio Grande do Sul (UFRGS) e na prática do Hospital de Clínicas de Porto Alegre (HCPA). ${ }^{3}$ Realizada até então de forma manual, a prescrição de enfermagem foi informatizada, em 2000, juntamente com a introdução dos diagnósticos de enfermagem segundo a Taxonomia I da North American Nursing Diagnosis Association (NANDA) associada ao referencial das Necessidades Humanas Básicas de Horta. ${ }^{4}$ Esta ferramenta tecnológica foi inicialmente introduzida no Centro de Terapia Intensiva (CTI) e, gradativamente, difundida para as demais unidades do HCPA. ${ }^{5}$ A principal estratégia na implantação do sistema informatizado visava a capacitar os enfermeiros para realização do raciocínio diagnóstico e utilização do sistema.

Os auxiliares e técnicos, membros da equipe de enfermagem, são os principais executores da prescrição realizada pelas enfermeiras. Contudo, poucos são os estudos encontrados que abordam a sua participação na metodologia da assistência ao paciente. Dentre eles, destaca-se um estudo realizado na mesma instituição da presente pesquisa, há cerca de duas décadas, cujo objetivo era conhecer como integrantes da equipe de enfermagem consideravam as prescrições de enfermagem, em relação ao conteúdo, execução e sugestões visando a seu aprimoramento. Participaram do mesmo 50 técnicos, auxiliares e os já extintos atendentes de enfermagem das unidades médico-cirúrgicas. ${ }^{6} \mathrm{Em}$ outro estudo, mais recente, enfocando a formação de técnicos de enfermagem por meio da análise dos planos de ensino de 26 cursos, foi constatada ênfase na realização de tarefas e atividades. Como competências gerais e especificas dos técnicos de enfermagem foi identificado o saber-fazer (54$59 \%)$, aprender a conhecer $(34-45 \%)$ e saber-ser
(1-7\%), retratando ainda a prevalência do saber fazer na educação profissionalizante, com tendência a fundamentar e instrumentalizar esse fazer com conhecimento científico. ${ }^{7}$

Em relação à normatização do exercício profissional quanto ao $\mathrm{PE}$, destaca-se a Lei $\mathrm{N}^{\circ}$ 7.498, de 25 de junho de 1986, pela qual foram determinados, como dever privativo do enfermeiro, o planejamento, organização, coordenação, execução e avaliação dos serviços, bem como a prescrição da assistência de enfermagem. Aos técnicos de enfermagem é atribuída a participação no planejamento da assistência, da orientação e supervisão do trabalho de enfermagem, bem como a execução de ações assistenciais. Quanto ao auxiliar de enfermagem, está estabelecida a sua participação em nível de execução simples, em processos de tratamento, cabendo-lhe observar, reconhecer e descrever sinais e sintomas, executar ações de tratamento simples, prestar cuidados de higiene e conforto ao paciente. ${ }^{8}$

Considerando-se a inserção do técnico e auxiliar na equipe de enfermagem, ao participar da avaliação do paciente, durante o cuidado prestado, é de fundamental importância que o seu conhecimento seja reconhecido e considerado para o desenvolvimento da Sistematização da Assistência de Enfermagem (SAE) concretizado por meio do PE. Alicerçado na legislação do exercício profissional e na priorização da SAE no Hospital Universitário da UFRGS, estabeleceu-se como objetivo desse estudo conhecer as reflexões de técnicos e auxiliares de enfermagem em relação ao PE, realizado do HCPA. Entende-se como reflexões as idéias e considerações manifestadas pelos profissionais sobre a temática.

\section{METODOLOGIA}

Trata-se de pesquisa com abordagem qualitativa exploratória descritiva, desenvolvida no HPCA, no primeiro semestre de 2006. Participaram auxiliares e técnicos de enfermagem, escolhidos de forma intencional, lotados em sete unidades de internação distintas e pertencentes aos três turnos de trabalho. Buscou-se contemplar pelo menos um sujeito de cada serviço e de todos os turnos, uma vez que o PE é utilizado em todas as unidades de internação da instituição.

Como critério de inclusão considerou-se possuir mais de seis meses de trabalho no HCPA. Foram excluídos trabalhadores de áreas onde a prescrição de enfermagem não era desenvolvida. 
A coleta de informações foi realizada por meio de entrevista semi-estruturada, na unidade e no turno de trabalho do entrevistado mediante concordância da enfermeira responsável. As questões que nortearam as entrevistas versaram sobre o seu conhecimento do PE e sobre qual a sua participação no mesmo. As entrevistas foram gravadas, excetuando-se uma, por solicitação do participante. O conteúdo de cada uma delas foi transcrito e analisado a partir da análise de conteúdo temática, seguindo as etapas preconizadas de pré-análise, analise do material e interpretação. ${ }^{9}$ Os sujeitos incluídos no estudo foram identificados pela letra " $\mathrm{e}$ " seguido de um número, de acordo com a seqüência das entrevistas, que foram realizadas entre abril e maio de 2006.

O projeto foi aprovado pelo Comitê de Ética em Pesquisa do HCPA, sob o protocolo $N^{\circ}$ 05-573, e todos os sujeitos assinaram o Termo de Consentimento Livre e Esclarecido anteriormente à entrevista.

\section{RESULTADOS}

Participaram, como sujeitos da pesquisa, 10 auxiliares e um técnico de enfermagem, esta diferença aconteceu porque na instituição somente as unidades de maior complexidade possuem técnicos de enfermagem contratados, sendo que nas quais não foram convidados os sujeitos a participarem da pesquisa. Os informantes tinham, em média, 39 anos de idade e 15 anos de profissão, sendo oito anos, em média, de trabalho no HCPA. Quanto à formação acadêmica, cinco possuíam formação parcial ou completa em nível superior, sendo dois em enfermagem.

Quanto à experiência anterior com PE, 5 $(45,45 \%)$ dos entrevistados já tinham contato com esta tecnologia, antes de ingressarem no HCPA. Os mesmos informaram que as enfermeiras examinavam os pacientes na admissão, evoluíam e uma delas prescrevia os cuidados para os pacientes.

A partir da análise das entrevistas emergiram cinco categorias, revelando as reflexões de auxiliares e técnicos sobre o PE desenvolvido no HCPA.

\section{Amplia a visão e organiza o cuidado}

O PE foi considerado importante e positivo por parte de auxiliares e técnicos, ampliando a visão que os profissionais têm acerca das condições do paciente: [...] eu acho que é muito bom porque é uma visão mais ampla. Quando vejo a prescrição de enfermagem vejo como é o meu paciente. Eu vejo as orientações e sei quando devem ser feitas [...] (e2).

[...] o PE dá uma visão mais ampla sobre o atendimento ao paciente, ao mesmo tempo em que permite focar algum problema ou situação específica [...] (e1).

Na admissão do paciente no ambiente hospitalar, a enfermeira realiza a anamnese e exame físi$\mathrm{Co}$, que é a primeira etapa do PE, visando conhecer o paciente, identificar suas necessidades e planejar os cuidados no âmbito de sua competência para solucioná-los ou minimizá-los. Esta investigação, também conhecida como avaliação inicial, compreende a coleta de informações abrangentes sobre aspectos que envolvem a saúde da pessoa, bem como dados para determinar a situação de uma condição específica, ${ }^{10}$ que pode tanto estar relacionada a aspectos biológicos, como emocionais e sociais. Por isso, durante o atendimento deve-se considerar o contexto no qual o paciente está inserido. ${ }^{11}$

Considerando a prescrição de enfermagem uma forma de delegar tarefas, a mesma precisa ter clareza e objetividade. ${ }^{12}$ Além disso, o PE, além de influenciar na organização/otimização da assistência, facilita o trabalho do auxiliar/técnico de enfermagem ao localizar no prontuário do paciente as informações concernentes à evolução do tratamento e aos cuidados a serem prestados. Esses aspectos foram mencionados por alguns informantes Outros também manifestaram que a prescrição de enfermagem dá visibilidade ao estado do paciente, facilitando o planejamento do seu turno de trabalho. Desse modo, fica configurada a importância da prescrição de enfermagem na delegação de tarefas e na qualificação da assistência por ela realizada.

Em diferentes momentos da entrevista foi possível constatar o conhecimento que os informantes possuem sobre o PE, tais como itens da prescrição e conhecimento das definições. Houve duas situações em que os informantes tinham estudado o PE no Curso de Graduação em enfermagem, embora todos demonstrassem algum conhecimento: [...] é a admissão, exame físico, prescrição de enfermagem, orientação ao paciente [...] (e9).

Os informantes também relataram alguns objetivos do PE: [...] meio de organizar e otimizar o atendimento ao paciente [...] (e1). [...] faz que a coisa não seja automatizada, [...] cada paciente precisa de um cuidado diferente [...] é importante porque consegue diferenciar o cuidado para cada paciente [...] (e8).

Os cuidados que constam no relato do informante e8 referem-se à prescrição de enfermagem, pois cada paciente precisa de um cuidado específi- 
co, de modo que as particularidades de cada indivíduo e as adaptações exigidas para o seu tratamento sejam contempladas em cada prescrição.

Analisando os relatos dos informantes, percebe-se que suas reflexões vão ao encontro da caracterização do PE, como um método sistemático, individualizado e humanizado da assistência de enfermagem. ${ }^{10}$

Durante as entrevistas não foi citada a etapa do diagnóstico de enfermagem. Esse fato pode ter ocorrido porque nem sempre os diagnósticos constam nas evoluções das enfermeiras e também não se encontram como item obrigatório do prontuário do paciente assim, os informantes podem não ter tido acesso a essa informação.

O conhecimento foi considerado como ponto positivo porque demonstra o interesse da equipe pela melhoria do paciente, visto que PE é uma metodologia científica que qualifica a assistência de enfermagem. E, como se observou, alguns informantes têm conhecimento dos itens, das características, e demonstram compreensão do PE. Em estudo anterior, relativo ao tema, foi verificado que o PE é uma forma de melhorar a assistência prestada ao paciente pela equipe de enfermagem. ${ }^{6}$ Em pesquisa recente, realizada com 70 auxiliares e 7 técnicos de enfermagem de um hospital do estado deSão Paulo, os resultados mostraram que os sujeitos valorizam a SAE por acreditarem que esta metodologia confere significado e consistência às suas atividades. ${ }^{13}$

Ao confrontarem-se dados teóricos e práticos sobre o PE, verificou-se que o mesmo está se desenvolvendo de maneira adequada, trazendo benefícios, tanto para o paciente atendido, que conta com cuidado qualificado e diferenciado, quanto para o técnico/auxiliar de enfermagem que está conseguindo otimizar seu tempo de serviço, tornando-o assim mais ágil e eficiente em suas atribuições.

\section{Aproxima a equipe de enfermagem}

O diálogo pode ser entendido como um processo de capacitação ao possibilitar que informações sejam transmitidas e idéias sejam traduzidas em ações, um processo de compreender, compartilhar mensagens enviadas e recebidas. Existem modos diferentes de comunicação, sendo mais freqüente a comunicação escrita, a comunicação face a face ou verbal, e comunicação não verbal. ${ }^{12,14-15}$

Em alguns grupos de trabalho ocorre troca de informações entre a enfermeira e o auxiliar/ técnico de enfermagem para elaboração da prescrição, assim como na etapa de anamnese e exame físico do paciente, favorecendo uma avaliação mais apurada e, aproximando a equipe.

[...] A gente faz uma avaliação juntas. A enfermeira pergunta quem está com aquele paciente, daí pergunta se tem curativo, ulcera de decúbito e conversamos como tem que fazer [...] (e2).

[...] tanto o enfermeiro já buscou informações quanto o auxiliar levou informações sobre o paciente, sendo que o enfermeiro foi bem receptivo [...] (e10).

Partindo dos relatos, observa-se que a comunicação verbal acontece de forma adequada em diferentes ocasiões. Comunicar-se, utilizando modalidade verbal e não verbal compreensíveis e ter um ouvir atento constituem-se elementos de suma importância no processo de liderar do enfermeiro. ${ }^{12,16}$ Considerando a equipe de enfermagem como um grupo de trabalho onde as pessoas interagem, o enfermeiro é o líder por ser sob sua responsabilidade que as atividades são realizadas. Sendo assim, é fundamental que haja uma comunicação adequada, destacando-se o ouvir atento e interessado, em que as palavras e as ações sejam coerentes. Ser respeitado na sua opinião, ao esclarecer itens que estão sem clareza ou que não foram compreendidos, contribui para uma melhora na satisfação e interesse em realizar o trabalho que está sendo proposto e possibilitando proximidade entre o grupo. Desta forma, equipes de trabalho que mantêm esse diálogo são usualmente mais produtivas, porque acreditam e se empenham no seu fazer, ou seja, no cuidado ao paciente.

\section{Favorece a continuidade dos cuidados}

Além da comunicação verbal, abordada anteriormente, a comunicação escrita por meio do registro no prontuário do paciente possibilita que todos os integrantes da equipe de saúde tenham acesso às informações, favorecendo a continuidade dos cuidados prestados: [...] uma continuidade do cuidado, daquilo que a enfermeira inicia; ela faz a prescrição, o exame físico, mas quem vai continuar aquilo ali? Então o bom do processo é que ele vai continuar o atendimento, o cuidado, o que vale aquela prescrição da enfermeira dentro da pasta, qualquer dúvida qualquer mudança ambos, enfermeiro e técnico ficam sabendo [...] (e4).

Os registros são um facilitador na unidade de ações sucessivas realizadas pela enfermagem, pois a cada turno, o técnico/auxiliar de enfermagem que está prestando assistência ao paciente pode ler a evolução anterior e conhecer os fatos importantes ocorridos com ele. A prescrição do enfermeiro, por sua vez, indica os cuidados que 
foram e deverão ser realizados, em um período de 24 horas, em prol da saúde do paciente no âmbito da enfermagem.

A linguagem utilizada foi um ponto destacado como positivo pelo informante e7, sendo dominada por todos os membros da equipe. A comunicação escrita deve ter clareza e uma linguagem compreensível, permitindo a documentação. ${ }^{12}$

Logo, os registros podem ser considerados como uma forma indispensável para a continuidade do tratamento do paciente, pois por meio da documentação das etapas do PE tem-se como realizar a avaliação dos resultados, considerando a evolução do paciente e, adaptando o plano de cuidados para cada fase do tratamento.

\section{Falhas na comunicação entre a equipe de enfermagem}

Como já destacado, a comunicação é de suma importância para o trabalho da enfermagem. Mesmo com as transformações sociais vivenciadas, a tecnologia ainda não substituiu a necessidade de orientar pessoas para alcançarem objetivos e metas. ${ }^{16}$ Assim a comunicação é ponto fundamental para manter uma equipe de trabalho atuando de forma integrada em prol do paciente, sendo que a sua falta pode gerar insatisfação.

[...] O Processo de enfermagem épositivo, porém, deve ser compartilhado com os demais membros da equipe, uma vez que os mesmos podem colaborar com informações preciosas em todos os estágios do Processo de enfermagem [...] (e1).

A reflexão do informante e1 demonstra que mesmo considerando positiva a utilização do PE, ocorre falta de diálogo, de troca de informações entre a sua equipe de trabalho. Salienta, ainda, que informações úteis para o tratamento ao paciente estão sendo perdidas por essa dificuldade da equipe.

[...] A enfermeira comunica as alterações na prescrição de enfermagem para dar continuidade ao cuidado, mas se não concordo com algo não tenho liberdade para falar com ela. Às vezes falta um pouquinho de receptividade porque ela vem com a coisa fechada [...] (e4).

O relato do informante 4 gera alguns questionamentos: será que o técnico/auxiliar de enfermagem que não concorda com a prescrição irá realizála? Ou irá registrar no prontuário a sua realização, conforme prescrita, contudo irá executá-la como pensa que deve ser feita? Os registros serão fidedignos com o ocorrido durante o cuidado prestado? E os aspectos éticos envolvidos nessa situação?
Em cada caso isso pode mudar, o técnico/ auxiliar de enfermagem pode agir de um jeito diferente, assim destaca-se que a preocupação deve estar centrada no ponto original, que é a comunicação, somente uma conversa em que ambos possam expor suas opiniões, quando é possivel acontecer troca de experiência e até uma comprovação por evidências, poder-se-á ter uma equipe, um trabalho em grupo onde todos buscam atingir um objetivo comum.

Também houve outros relatos que demonstraram essa falta de diálogo, a exemplo do informante: [...] A conduta das enfermeiras conosco poderia ser melhorada porque às vezes acontece uma certa prepotência, a palavra delas é a última [...] (e4).

Além da falta de diálogo, essa fala refere que as regras são impostas. Ressalta-se a relevância do papel da enfermeira, como organizadora e coordenadora das atividades assistenciais, desenvolver algumas competências como a comunicação e liderança, competências essas incluídas nas Diretrizes Curriculares Nacionais como essenciais na formação do enfermeiro. A liderança revela o processo pelo qual um grupo é induzido a dedicar-se aos objetivos defendidos pelo líder, envolvendo empatia, responsabilidade e habilidade na tomada de decisões e comunicação. ${ }^{15}$ Assim, cabe ao enfermeiro utilizar essas competências para mobilizar a equipe em torno de um objetivo, valendo-se do potencial de todos os participantes na organização do serviço, o que contribui para a satisfação no trabalho e principalmente a melhoria na assistência ao paciente, que é o foco principal das ações de enfermagem.

\section{Necessidade de reavaliação da prescrição dos cuidados de enfermagem}

A prescrição de enfermagem foi um aspecto bastante comentado durante as entrevistas assim, emergindo pontos considerados problemáticos: [...] A prescrição deveria ser mais enxuta, e as rotinas não precisavam estar dentro dela (e6).

A extensão das prescrições foi uma questão relatada, e que faz refletir sobre a sua ocorrência. Há cuidados que estão sendo prescritos duplamente ou de forma inadequada? As "rotinas" poderiam ser excluídas das prescrições? Contudo, é importante considerar que muitos pacientes, com tratamentos complexos, necessitam de um grande número de cuidados.

Durante as entrevistas, questionou-se sobre o entendimento dessas "rotinas", obtendo a informa- 
ção de que seriam cuidados prestados para todos os pacientes, como exemplo, a verificação de sinais vitais e o banho. A prescrição serve de roteiro para realização das atividades de enfermagem, além disso, ajuda no ensino dos alunos de enfermagem a tomar decisões, e a partir dela pode-se determinar o custo dos serviços prestados por enfermeiros e planejar os recursos necessários para a prática da enfermagem. ${ }^{17}$ Neste sentido, a omissão dos itens denominados "rotinas" poderia invalidar esses objetivos. Além disso, há no HCPA um grande número de estudantes, do nível técnico e de graduação, que utilizam o hospital como campo de estágio, e a prescrição de cada um dos cuidados, rotineiros ou não, auxilia na sua formação.

Os relatos de auxiliares e técnicos de enfermagem convergem com a opinião das enfermeiras. Um aspecto a ser melhorado, segundo as próprias enfermeiras do HCPA, refere-se à prescrição de enfermagem ser rotineira, contendo aquilo que é padronizado e não os cuidados específicos de cada paciente. Elas alegam que os cuidados de rotina deveriam estar internalizados nos auxiliares e técnicos de enfermagem, e apenas os cuidados individuais e prioritários de cada paciente deveriam constar na prescrição, pois, espera-se que a enfermeira tenha conhecimento e uma visão mais profunda dos problemas dos pacientes. ${ }^{5}$ Essa queixa já havia sido manifestada pelo pessoal auxiliar de enfermagem do HCPA, na década de 80, alegando como uma desvantagem da prescrição de enfermagem conter as rotinas já conhecidas. ${ }^{6}$

$\mathrm{O}$ relato a seguir sugere fragilidades na avaliação do paciente, realizada pela enfermeira, e na atualização da prescrição: [...] às vezes acho que a prescrição é muito mecânica é colocada para repetir, às vezes é superficial (e1).

Esses aspectos foram destacados em estudo realizado no HCPA, no qual as enfermeiras relataram que algumas de suas colegas prescrevem o tratamento dos pacientes sem avaliá-los previamente, ou não atualizam suas prescrições, quando necessário. Um exemplo citado refere-se a uma prescrição que continha cuidados com soroterapia quando esta já havia sido suspensa, há quatro dias. ${ }^{5}$ Esse fato demonstra que, tanto auxiliares e técnicos de enfermagem, quanto enfermeiras, percebem essa falha na prescrição.

Os problemas na prescrição de enfermagem relatados por alguns informantes evidenciam dificuldades de comunicação, gerando insatisfação no trabalho. Como proposta de solução, sugerese criar espaços de diálogo entre os membros da equipe de enfermagem onde aspectos relativos ao cuidado dos pacientes e contidos na prescrição fossem discutidos.

\section{CONSIDERAÇÕES FINAIS}

As categorias: amplia a visão e organiza o cuidado, aproxima a equipe de enfermagem e favorece a continuidade dos cuidados englobam elementos reconhecidos como positivos, além daqueles identificados como importantes para a aplicação e desenvolvimento do PE. Por outro lado, nas categorias: falhas na comunicação entre a equipe de enfermagem e necessidade de reavaliação da prescrição dos cuidados de enfermagem observaram-se relatos de descontentamento e os elementos que os entrevistados identificaram como problemáticos para a aplicação e desenvolvimento do PE.

Considera-se que os auxiliares e técnicos ocupam importante espaço no desenvolvimento do PE, pois tornam efetiva a prescrição de enfermagem, convertendo-a em ações práticas, com repercussões favoráveis à saúde do paciente.

A opinião dos auxiliares/técnicos de enfermagem sobre o PE demonstra como se eles se sentem inseridos, pois além de aceitarem o desenvolvimento do mesmo, também conseguem identificar elementos que auxiliam no seu trabalho, trazendo benefícios para o paciente e também para os funcionários que estão executando os cuidados de enfermagem. O reconhecimento que o pessoal de enfermagem demonstrou sobre a avaliação que a enfermeira faz do paciente serve de incentivo a continuar buscando conhecimento para melhorar cada vez mais o PE.

Os auxiliares/técnicos de enfermagem demonstraram que conheciam quase todas etapas do PE, e esse conhecimento ocorreu durante o exercício de profissional. Desta forma sugere-se que os professores de cursos técnicos de enfermagem incluam no currículo o conteúdo do PE na formação desses profissionais.

O ponto que gerou mais polêmica foi a comunicação, refletindo como o grupo de trabalho interage. Alguns informantes apontaram o diálogo como positivo do PE, pois através dele conseguiam realizar uma avaliação mais apurada do paciente e, porque os auxiliares/técnicos de enfermagem, juntamente com a enfermeira, podiam discutir a situação enfrentada pelo paciente e chegar a um consenso, onde era oportunizado exporem sua opinião. Porém, outro grupo trazia o discurso inverso, 
evidenciando aspectos a serem melhorados no PE. Relataram que a enfermeira impunha sua conduta, não havia diálogo e a comunicação se dava em uma única direção, para informar alterações de cuidados com o paciente.

Este fato gera preocupação, uma vez que a falta de efetividade na comunicação pode prejudicar a qualidade dos cuidados prestados e das informações geradas pelos auxiliares/técnicos de enfermagem. Questiona-se se em um ambiente de trabalho, onde há reduzida valorização do conhecimento e experiência do trabalhador, o mesmo irá realizar as tarefas com as quais discorda ou que acha desnecessárias. Isso envolve aspectos éticos que merecem ser refletidos por todos os membros da equipe de trabalho.

Uma limitação do estudo foi a escassa literatura encontrada sobre a temática PE relacionada ao auxiliar e técnico de enfermagem, parecendo, em um primeiro momento, desatualizada. No entanto, observou-se que algumas opiniões presentes havia duas décadas eram ainda atuais. As vantagens e alguns problemas como, por exemplo, cuidados rotineiros na prescrição de enfermagem permanecem inalterados. $\mathrm{O}$ que se propõe é promover espaços de discussão em torno da prescrição de enfermagem, em que cada equipe possa chegar a um consenso razoável, determinando combinações que tragam satisfação para a equipe.

Com o término desse estudo obtiveram-se algumas respostas e novas indagações. Enfatizase a importância da comunicação para o trabalho e a expressão da escuta, respeitando as idéias e indagações sem juízo prévio. Analisando as considerações de auxiliares e técnicos de enfermagem sobre o PE praticado no HCPA pôde-se perceber que eles têm muito a contribuir, e que estão junto com as enfermeiras em prol da melhoria do cuidado prestado ao paciente.

O foco da pesquisa foi o PE, mas no decorrer das entrevistas foi possível perceber que esta temática serviu como pano de fundo para outras questões envolvidas no dia-a-dia do trabalho em equipe, tais como liderança e comunicação. Depreende-se daí que se os integrantes de uma mesma equipe não trabalharem em harmonia, isto se reflete no cuidado como um todo, desde o planejamento, sua implementação e avaliação, que é o PE.

Diante do exposto sugere-se fomentar educação em serviço para a equipe de enfermagem, tendo como foco o PE, dando-se ênfase à comunicação. Tendo em vista que a etapa do diagnóstico de enfermagem não é conhecida pelos informantes, propõe-se que seja um item mais trabalhado na equipe de enfermagem e sua documentação estimulada no prontuário do paciente. Acrescenta-se que os problemas devem ser discutidos, pois somente o dialogo fará com que o PE continue se desenvolvendo.

Tendo em vista que o tema PE é ainda pouco explorado junto aos integrantes técnicos da equipe de enfermagem e diante da relevância do assunto, novos estudos podem ser desenvolvidos, buscando melhorias a esse processo e satisfação profissional à equipe.

\section{REFERÊNCIAS}

1 Garcia TR, Nóbrega MM. Processo de enfermagem e os sistemas de classificação dos elementos da prática profissional: instrumentos metodológicos e tecnológicos do cuidar. In: Santos I, Figueiredo NMA, Padilha MICS, Souza SROS, Machado WCA, Cupello AJ. Enfermagem Assistencial no ambiente hospitalar: realidade, questões, soluções. São Paulo (SP): Atheneu; 2005. p.37-63.

2 Rossi LA, Casagrande LDR. Processo de enfermagem: a ideologia da rotina e a utopia do cuidado individualizado. In: Cianciarullo TI, Gualda DMR, Melleiro MM, Anabuki MH. Sistema de assistência de enfermagem: evolução e tendências. São Paulo (SP): Ícone; 2001. p.41-62.

3 Almeida MA. Sistematização da assistência de enfermagem na formação do enfermeiro. In: Chianca TC, Rocha AM, Pimentel MA, organizadoras. Anais do 7o Simpósio Nacional de Diagnósticos de Enfermagem; 2004 Maio 29-Jun 01; Belo Horizonte, Brasil. Belo Horizonte (MG): ABEn-Seção MG; 2004. p.88-97.

4 Benedet SA, Bub MBC. Manual de diagnóstico de enfermagem: uma abordagem baseada na Teoria das necessidades humanas e na classificação diagnóstica da NANDA. 2a ed. Florianópolis (SC): Bernúncia Ed.; 2001.

5 Elizalde AC, Almeida MA. Percepções de enfermeiras de um hospital universitário sobre a implantação dos diagnósticos de enfermagem. Rev. Gaúch. Enferm. 2006 Dez; 27 (4): 563-73.

6 Cogo ALP, Borba EL, Ferreira MAS, Piazza MGC, Scholem VGS. Percepção de enfermagem: percepção do pessoal auxiliar de enfermagem de unidades médico-cirúrgicas em um hospital de ensino. Rev. Gaúch. Enferm. 1986 Jan; 7 (1): 61-77.

7 Kobayashi RM, Leite MMJ. Formação de competências administrativas do técnico de enfermagem. Rev. Latino-Am. Enfermagem. 2004 Mar-Abr; 12 (2): 221-7. 
8 Brasil. Lei No 7.498, de 25 de junho de 1986. Dispõe sobre a regulamentação do exercício da Enfermagem, e dá outras providências. Brasília (DF): COFEN, 2006 [acesso em 2007 Maio 22]. Disponível em: http:/ / www.portalcofen.gov.br/2007/materias. asp?ArticleID=22\&sectionID $=35$

9 Bardin L. Análise de conteúdo. 3a ed. Portugal (PT): Edições 70; 2004.

10 Alfaro-Lefèvre R. Aplicação do processo de enfermagem: promoção do cuidado colaborativo. 5a ed. Porto Alegre (RS): ARTMED; 2005.

11 Silva WV, Nakata S. Comunicação: uma necessidade percebida no período pré-operatório de pacientes cirúrgicos. Rev. Bras. Enferm. 2005 Nov-Dez; 6 (58): 673-6.

12 Marquis BL, Huton C J. Administração e liderança em enfermagem: teoria e aplicação. 2a ed. Porto Alegre (RS): Artes Médicas Sul; 1999.
13 Ramos LAR. Sistematização da assistência de enfermagem: um estudo com auxiliares e técnicos de enfermagem [dissertação]. Ribeirão Preto (SP): USP/EERP; 2007.

14 Santos KMAB, Silva MJP. Comunicação entre líderes e liderados: visão dos enfermeiros. Rev. Escola Enferm. USP. 2003 Jun; 2 (37): 97-108.

15 Peres AM, Ciampone MHT. Gerência e competências gerais do enfermeiro. Texto Contexto Enferm. [on line]. 2006 Jul-Set; 15 (3): 492-9 [acesso em 2007 Maio 22]. Disponível em: http://www.textoecontexto. ufsc.br/viewarticle.php?id=199

16 Balsanelli AP, Cunha ICKO. Liderança no contexto da enfermagem. Rev. Escola Enferm. USP. 2006 Mar; 1 (40): 117-22.

17 McCloskey JC, Bulechek GM. Classificação das Intervenções de Enfermagem (NIC). 3a ed. Porto Alegre (RS): Artmed; 2004. 